\title{
Volumetric and Ultrasonic Study of Mixtures of Benzyl Alcohol with 1-Propanol, 2-Propanol, and 1,2-Propandiol, 1,3-Propandiol and T-butanol
}

\author{
Fatemeh Fadaei Nobandegani ${ }^{1, \text { *, Abouzar Roeintan }}{ }^{2}$ \\ ${ }^{1}$ Department of Food Science and Thecnology, Fasa University, Fasa, Iran \\ ${ }^{2}$ Department of Chemistry, Emam Hossein University, Tehran, Iran \\ Email address: \\ Fatima_Fadaei_84@Yahoo.Com (F. F. Nobandegani) \\ ${ }^{*}$ Corresponding author
}

\section{To cite this article:}

Fatemeh Fadaei Nobandegani, Abouzar Roeintan. Volumetric and Ultrasonic Study of Mixtures of Benzyl Alcohol with 1-Propanol, 2Propanol, and 1,2-Propandiol, 1,3-Propandiol and T-butanol. American Journal of Mechanics and Applications.

Vol. 7, No. 4, 2019, pp. 88-100. doi: 10.11648/j.ajma.20190704.12

Received: June 22, 2019; Accepted: July 15, 2019; Published: January 8, 2020

\begin{abstract}
Densities and speeds of sound for five binary mixtures of benzylalcohol with 1,3-propandiol, 1- propanol, 2 propanol, 1,2-propandiol and phenylethnol were measured over the entire range of composition and at five temperatures from $298.15 \mathrm{~K}$ to $323.15 \mathrm{~K}$ at $5 \mathrm{~K}$ interval and atmospheric pressure using a vibrating u-tube densimeter (DSA 5000). Besides, the densities for pure compounds in the above-mentioned temperature range were measured. The experimental densities were used to calculate the excess molar volumes, isentropic compressibility changes, the excess thermal expansion coefficients, and the excess partial molar volumes at infinite dilution, The results have been used to discuss the nature and strength of intermolecular interactions in these mixtures. The calculated excess and deviations quantities are correlated with the third-order Redlich-Kister equation. As a final work we modeled the experiment results by using TM and PR EOSs. This is clear that the results with TM EOS are more acceptable than PR EOS. TM and PR EOS can successfully predict density and excess molar volume. And are unable to predict speed of sound.
\end{abstract}

Keywords: Alcohols, Binary Mixtures, Excess Properties, Density, Modeling, Speed of Sound

\section{Introduction}

Thermodynamic properties for pure and mixtures of liquids especially alcohols have both practical and theoretical interest; They have to be known to design industrial processes properly. They can be used to develop models that allow us to predict other properties. Finally, they can also reveal the existence of specific molecular interactions. In recent years, measurements of thermodynamic and transport properties have been adequately employed in understanding the nature of molecular systems and physicochemical behavior in liquid mixtures Despite the extensive studies on binary mixtures of alcohols [1-7], so far relatively less attention has been given to the mixtures containing an aromatic alcohol [8]. The mixtures of benzyl alcohol with aliphatic alcohols are interesting to study because of the possibility of weak $\pi \ldots \mathrm{H}$ bonding, in addition to the hydrogen bonding between unlike molecules. The weak $\pi$...H bonding of aromatic rings with proton donors appears to play an important role in the structure of certain bimolecular [9]. Yeh et al. [10] reported thermophysical properties of the binary mixtures 2-phenylethanol+2propanol, 2-phenylethanol+benzylalcohol, at $\mathrm{T}=(298.15$, 308.15, and 318.15) K. Huang et al. [11]. Sastry et al reported Densities, excess molar and partial molar volumes for water + 1-butyl- or, 1-hexyl- or, 1-octyl-3methylimidazolium halide room temperature ionic liquids at $\mathrm{T}=(298.15$ and 308.15) $\mathrm{K}$ [12]. Gyan parkesh et al investigated thermodynamic, thermophysical and partial molar properties of liquid mixtures of diethylenediamine with alcohols at 293.15 to 313.15 K [13] and Sk. Md Nayeem et al investigated thermoacoustic, Volumetric, and Viscometric in Binary Liquid System of Cyclohexanone with Benzyl Benzoate at T $=308.15,313.15$, and 318.15 K [14]. 
In another search we use butanol and investigated thire thermodynamic properties [15-17] and in continues in present study, we report densities $(\rho)$ and speeds of sound $(u)$ for the binary mixtures of benzylalcohol with 1-propanol, 2propanol, 1,2-propandiol, and 1,3-propandiol and t-butanol at $\mathrm{T}=(298.15,303.15,308.15,313.15$ and 318.15$) \mathrm{K}$ and in the entire composition range and atmospheric pressure using a vibrating u-tube densimeter (DSA 5000). The experimental values of $\rho$ and $u$ were used to calculate the excess molar volumes $\left(V_{m}^{E}\right)$, deviation in isentropic compressibility $\left(\Delta \kappa_{S}\right)$, excess thermal expansion coefficient $\left(\alpha_{P}^{E}\right)$, infinite partial molar volumes $\left(\bar{V}_{i}^{\infty}\right)$, and excess partial molar volume $\left(\bar{V}_{i}^{E, \infty}\right)$ of the components at infinite dilution. The variation of these parameters with the composition and temperature of the mixtures have been discussed in terms of molecular interactions. Additionally, the density and excess molar volumes of the studied alcohols (pure and mixtures) were modeled with Tao-Mason (TM) and Peng-Robinson (PR) equations of state.

\section{Experimental}

1-propanol, 2-propanol, 1,3-propandiol (mass fraction > 98\%, Merck), 1,2-propandiol (mass fraction $>98 \%$, Merck) benzyl alcohol (mass fraction $\geq 99.5 \%$, Merck) and t-butanol (mass fraction $\geq 99 \%$, Merck) were used without further purification.

Densities and speeds of sound for pure liquids and their binary mixtures were measured using an Anton Paar DSA 5000 vibrating $\mathrm{u}$-tube densimeter and sound analyzer, with a certified precision of $\pm 1 \cdot 10^{-6} \mathrm{~g}^{*} \mathrm{c}^{\mathrm{m}-3}$ and $\pm 0.1 \mathrm{~m}^{*} \mathrm{~s}-1$, respectively. This analyzer automatically corrects the influence of viscosity on the measured density. The DSA 5000 simultaneously determines two physically independent properties within one sample. The instrument is equipped with a density cell and a sound speed cell combining the known oscillating u-tube method with a highly accurate measurement of sound speed. Two integrated Pt 100 platinum thermometers together with Peltier elements provide a good precision in temperature control internally $(T \pm 0.001 \mathrm{~K})$. The apparatus was checked once a day and calibration was carried out with double distilled water and dry air under atmospheric pressure. Before the measurements, all the components were partially degassed $(20 \mathrm{~min})$ using an ultrasound (MISONIX Ultrasonic Liquid Processors). Solutions were prepared by mass using an analytical balance with a precision of $\pm 1 \cdot 10^{-4} \mathrm{~g}$ and kept in sealed flasks. The experimental uncertainties in the density and speed of sound are estimated to be better than $\pm 5 \times 10^{-6} \mathrm{~g} \mathrm{c}^{\mathrm{m}-3}$ and \pm 0.05 $\mathrm{m}^{\mathrm{s}-1}$, respectively. Precautions were taken in order to minimize evaporation losses during storage and preparation of the solutions.

\section{Results and Discussion}

The experimental densities and speeds of sound of pure liquids of this work appear in Table (1). Our results agree well with those reported in literature [7]. This agreement gives a verification of the results obtained by the densimeter. After preparation of solutions, the densities and speeds of sound for all five binary mixtures of benzyl alcohol with 1propanol, 2-propanol, 1,3-propandiol, 1,2-propandiol and phenylethanol over the entire composition range and at five different temperatures were measured using u-tube vibrating densimeter. The results are presented in Tables (2) - (6).

Table 1. Densities, $\rho$, and speeds of sound, $u$, for the pure components.

\begin{tabular}{|c|c|c|}
\hline$T / \mathbf{K}$ & $\rho /\left(\mathbf{g} \cdot \mathbf{c m}^{-3}\right)$ & $u /\left(\mathbf{m} \cdot \mathbf{s}^{-1}\right)$ \\
\hline \multicolumn{3}{|c|}{ benzylalcohol } \\
\hline 298.15 & 1.04129 & \\
\hline 303.15 & 1.03743 & 1509.43 \\
\hline 308.15 & 1.03355 & 1493.7 \\
\hline 313.15 & 1.02966 & 1478.25 \\
\hline 318.15 & 1.02575 & 1462.82 \\
\hline \multicolumn{3}{|c|}{ 1-propanol } \\
\hline 298.15 & 0.79973 & 1205.88 \\
\hline 303.15 & 0.79570 & 1189.02 \\
\hline 308.15 & 0.79163 & 1172.04 \\
\hline 313.15 & 0.78755 & 1155.16 \\
\hline 318.15 & 0.78336 & 1138.34 \\
\hline \multicolumn{3}{|c|}{ 2-propanol } \\
\hline 298.15 & 0.78128 & 1139.36 \\
\hline 303.15 & 0.77701 & 1121.96 \\
\hline 308.15 & 0.77265 & 1104.33 \\
\hline 313.15 & 0.76821 & 1086.63 \\
\hline 318.15 & 0.76367 & 1068.84 \\
\hline \multicolumn{3}{|c|}{ 1,2-propandiol } \\
\hline 298.15 & 1.03250 & 1508.82 \\
\hline 303.15 & 1.02879 & 1494.95 \\
\hline 308.15 & 1.02504 & 1480.89 \\
\hline 313.15 & 1.02125 & 1466.77 \\
\hline 318.15 & 1.01743 & 1452.62 \\
\hline \multicolumn{3}{|c|}{ 1, 3propandiol } \\
\hline 298.15 & 1.05206 & 1625.97 \\
\hline 303.15 & 1.04889 & 1614.65 \\
\hline 308.15 & 1.04571 & 1603.23 \\
\hline 313.15 & 1.04250 & 1591.95 \\
\hline 318.15 & 1.03928 & 1580.78 \\
\hline \multicolumn{3}{|c|}{ phenylethanol } \\
\hline 298.15 & 1.01615 & 1526.09 \\
\hline 303.15 & 1.01239 & 1510.48 \\
\hline 308.15 & 1.00861 & 1494.66 \\
\hline 313.15 & 1.00475 & 1479.17 \\
\hline 318.15 & 1.00093 & 1463.25 \\
\hline
\end{tabular}

\subsection{Excess Molar Volume}

Excess molar volumes, $V_{m}^{E}$, were calculated from density data according to the following equation:

$$
V_{m}^{E}=\sum_{i=1}^{2} x_{i} M_{i}\left(\rho^{-1}-\rho_{i}^{-1}\right)
$$

where $x_{i}, M_{i}$, and $\rho_{i}$ are the mole fraction, the molar mass and the density of the pure component $i$ respectively, $\rho$ is the density of the mixtures, The outcome of the computations has been listed in Table (2) - (6). Figures (1) (a) - (5) (a) shows these results for the excess molar volumes for the given binary mixtures versus the mole fraction at different temperatures. Considering $V_{m}^{E}$ s shown in Tables (2) - (6) as well as Figures (1) (a)-(5) (a), it is evident that $V_{m}^{E}$ is negative for the all binary systems of benzyl alcohol +1 , 3 propandiol, 1, 2propandiol, 1-propanol, 2-propnol and tbutanol over the entire composition range and all temperatures. Moreover, a close inspection of the $V_{m}^{E}$ s curves 
indicates that the curve skew occurs toward $x_{1}=1$ at the sequence of mixtures of benzyl alcohol with1-propanol, 2propanol, 1, 2 propandiol, 1,3-propandioldiol, and t-butanol. It means that for the mixture benzylalcohol with 1-propanol minimum occurs at $\mathrm{x}_{1}=0.6$ while for the other mixtures benzylalcohol with the other alcohol the minimum occurs between $\mathrm{x}_{1}=0.4$ and 0.6 , respectively. This behavior may be related to the difference in size and shape of the mixture partners. The negative minus of $V_{m}^{E}$ indicates a decrease in the overall volume of the mixture with respect to pure ones. The contraction of the volume can be attributed to strong interactions (mainly due to $\mathrm{H}$-bonding) between unlike molecules. On the other hand, the positive $V_{m}^{E} \mathrm{~s}$ values demonstrate a loose packing of molecules in the mixture, that is, a dilatation.

Table 2. Densities, $\rho$, speeds of sound, $u$, excess molar volumes, $V_{m}^{E}$, deviation in isentropic compressibility's, $\Delta \kappa_{s}$, and excess thermal expansion coefficients $\alpha_{P}^{E}$ as functions of mole fraction, $x_{1}$ of glycerol for $\{$ benzyl alcohol (1) +1,2-propanol (2)\} mixtures at the temperatures (298.15 to 318.15$)$ K.

\begin{tabular}{|c|c|c|c|c|c|}
\hline$x_{1}$ & $\rho / \mathrm{g}^{*} \mathrm{~cm}^{-3}$ & $u / \mathrm{m}^{*} \mathrm{~s}^{-1}$ & $V_{m}^{E} / \mathrm{cm}^{3^{*}} \mathrm{~mol}^{-1}$ & $\Delta \kappa_{S} / \mathrm{TPa}^{-1}$ & $10^{4} \cdot \alpha_{P}^{E} / \mathrm{K}^{-1}$ \\
\hline \multicolumn{6}{|c|}{$T=298.15 \mathrm{~K}$} \\
\hline 0.0000 & 1.03272 & 1505.88 & 0.000 & 0.00 & 0.000 \\
\hline 0.1034 & 1.044602 & 1550.43 & -0.206 & -22.42 & -0.025 \\
\hline 0.2049 & 1.065623 & 1589.17 & -0.369 & -32.60 & -0.880 \\
\hline 0.3051 & 1.07363 & 1626.96 & -0.492 & -37.30 & -0.423 \\
\hline 0.4062 & 1.09827 & 1661.86 & -0.557 & -39.02 & -0.453 \\
\hline 0.5042 & 1.105712 & 1694.5 & -0.577 & -39.90 & -0.463 \\
\hline 0.6032 & 1.138194 & 1722.94 & -0.592 & -40.63 & -0.297 \\
\hline 0.8057 & 1.207981 & 177940 & -0.547 & -37.36 & -0.098 \\
\hline 0.9034 & 1.225682 & 1703.72 & -0.253 & -27.55 & -0.232 \\
\hline 1.0000 & 1.34129 & 1725.13 & 0.000 & 0.00 & 0.000 \\
\hline \multicolumn{6}{|c|}{$T=303.15 \mathrm{~K}$} \\
\hline 0.0000 & 1.035697 & 1371.68 & 0.000 & 0.00 & 0.000 \\
\hline 0.1034 & 1.04057 & 1507.83 & -0.205 & -24.35 & -0.062 \\
\hline 0.2049 & 1.051161 & 1543.3 & -0.489 & -36.01 & -0.645 \\
\hline 0.3051 & 1.070618 & 1581.84 & -0.600 & -42.29 & -0.371 \\
\hline 0.5042 & 1.10676 & 1680.88 & -0.625 & -45.46 & -0.490 \\
\hline 0.6032 & 1.144239 & 1748.99 & -0.651 & -44.03 & -0.352 \\
\hline 0.7048 & 1.15486 & 1739.41 & -0.575 & -43.54 & -0.136 \\
\hline 0.8057 & 1.204066 & 1861.34 & -0.455 & -42.16 & -0.118 \\
\hline 0.9034 & 1.261794 & 1992.34 & -0.280 & -29.79 & -0.180 \\
\hline 1.0000 & 1.33743 & 2002.17 & 0.000 & 0.00 & 0.000 \\
\hline \multicolumn{6}{|c|}{$T=308.15 \mathrm{~K}$} \\
\hline 0.0000 & 1.038626 & 1372.04 & 0.000 & 0.00 & 0.000 \\
\hline 0.1034 & 1.056496 & 1416.78 & -0.254 & -26.59 & -0.095 \\
\hline 0.2049 & 1.07562 & 1456.1 & -0.501 & -39.99 & -0.426 \\
\hline 0.3051 & 1.086574 & 1493.98 & -0.557 & -46.33 & -0.315 \\
\hline 0.4062 & 1.093243 & 1528.95 & -0.683 & -49.36 & -0.510 \\
\hline 0.5042 & 1.237751 & 1561.92 & -0.634 & -50.45 & -0.506 \\
\hline 0.6032 & 1.310249 & 1592.53 & -0.665 & -49.69 & -0.399 \\
\hline 0.7048 & 1.390896 & 1620.82 & -0.583 & -47.89 & -0.160 \\
\hline 0.8057 & 1.50129 & 1647.37 & -0.454 & -44.43 & -0.129 \\
\hline 0.9034 & 1.597885 & 1672.07 & -0.327 & -31.61 & -0.125 \\
\hline
\end{tabular}

Table 2. Continued.

\begin{tabular}{|c|c|c|c|c|c|}
\hline$x_{1}$ & $\rho / \mathrm{g}^{*} \mathrm{~cm}^{-3}$ & $u / m^{*} s^{-1}$ & $V_{m}^{E} / \mathrm{cm}^{3^{*}} \mathrm{~mol}^{-1}$ & $\Delta \kappa_{S} / \mathrm{TPa}^{-1}$ & $10^{4} \cdot \alpha_{P}^{E} / K^{-1}$ \\
\hline \multicolumn{6}{|c|}{$T=313.15 \mathrm{~K}$} \\
\hline 0.0000 & 1.047518 & 1355.16 & 0.000 & 0.00 & 0.000 \\
\hline 0.1034 & 1.052387 & 1400.05 & -0.250 & -29.40 & -0.139 \\
\hline 0.3051 & 1.082496 & 1477.55 & -0.564 & -52.16 & -0.263 \\
\hline 0.4062 & 1. 09183 & 1512.59 & -0.657 & -55.01 & -0.526 \\
\hline 0.5042 & 1.99372 & 1545.68 & -0.684 & -56.23 & -0.511 \\
\hline 0.6032 & 1.136238 & 1576.43 & -0.593 & -55.01 & -0.449 \\
\hline 0.7048 & 1.20691 & 1604.84 & -0.505 & -52.51 & -0.110 \\
\hline 0.8057 & 1.36174 & 1631.58 & -0.387 & -47.83 & -0.146 \\
\hline 0.9034 & 1.413963 & 1656.39 & -0.251 & -33.12 & -0.079 \\
\hline 1.0000 & 1.62966 & 1678.25 & 0.000 & 0.00 & 0.000 \\
\hline \multicolumn{6}{|c|}{$T=318.15 \mathrm{~K}$} \\
\hline 0.0000 & 1.03359 & 1338.34 & 0.000 & 0.00 & 0.000 \\
\hline 0.1034 & 1.048231 & 1383.4 & -0.226 & -32.39 & -0.180 \\
\hline 0.2049 & 1.069338 & 1423.04 & -0.450 & -49.11 & 0.018 \\
\hline 0.3051 & 1.078383 & 1461.2 & -0.565 & -57.30 & -0.206 \\
\hline 0.5042 & 1.09649 & 1529.59 & -0.673 & -62.85 & -0.517 \\
\hline 0.6032 & 1.19199 & 1560.45 & -0.628 & -60.36 & -0.503 \\
\hline
\end{tabular}




\begin{tabular}{llllll}
\hline $\boldsymbol{x}_{\mathbf{1}}$ & $\boldsymbol{\rho} / \mathbf{g}^{*} \mathbf{c m}^{-\mathbf{3}}$ & $\boldsymbol{u} / \mathbf{m}^{*} \mathbf{s}^{-\mathbf{1}}$ & $\boldsymbol{V}_{\boldsymbol{m}}^{E} / \mathbf{c m}^{\mathbf{3}^{*} \mathbf{m o l}} \mathbf{l}^{-\mathbf{1}}$ & $\Delta \boldsymbol{\kappa}_{\boldsymbol{S}} / \mathbf{T P \mathbf { T P } ^ { - 1 }}$ & $\mathbf{1 0}^{\mathbf{4}} \cdot \boldsymbol{\alpha}_{P}^{E} / \mathbf{K}^{-1}$ \\
\hline 0.7048 & 1.3029 & 1588.99 & -0.505 & -57.16 & -0.055 \\
0.8057 & 1.52199 & 1615.93 & -0.391 & -51.35 & -0.158 \\
0.9034 & 1.6002 & 1640.85 & -0.254 & -35.00 & -0.039 \\
1.0000 & 1.72575 & 1662.82 & 0.000 & 0.00 & 0.000 \\
\hline
\end{tabular}

Table 3. Densities, $\rho$, speeds of sound, $u$, excess molar volumes, $V_{m}^{E}$, deviation in isentropic compressibility, $\Delta \kappa_{s}$, and excess thermal expansion coefficients $\alpha_{P}^{E}$ as functions of mole fraction, $x_{1}$ of glycerol for $\{$ benzyl alcohol (1) + 1-propanol (2)\} mixtures at the temperatures (298.15 to 318.15) K.

\begin{tabular}{|c|c|c|c|c|c|}
\hline$x_{1}$ & $\rho / \mathrm{g}^{*} \mathrm{~cm}^{-3}$ & $u / m^{*} s^{-1}$ & $V_{m}^{E} / \mathrm{cm}^{3^{*}} \mathrm{~mol}^{-1}$ & $\Delta \kappa_{S} / \mathrm{TPa}^{-1}$ & $10^{4} \cdot \alpha_{P}^{E} / \mathrm{K}^{-1}$ \\
\hline \multicolumn{6}{|c|}{$T=298.15 \mathrm{~K}$} \\
\hline 0.0000 & 0.79972 & 1205.88 & 0.000 & 0.00 & 0.000 \\
\hline 0.1034 & 0.834602 & 1250.43 & -0.196 & -21.50 & -0.016 \\
\hline 0.2049 & 0.865623 & 1289.17 & -0.339 & -31.62 & -0.863 \\
\hline 0.3051 & 0.89463 & 1326.96 & -0.482 & -37.00 & -0.403 \\
\hline 0.4062 & 0.921272 & 1361.86 & -0.537 & -38.55 & -0.446 \\
\hline 0.5042 & 0.945712 & 1394.5 & -0.567 & -39.51 & -0.473 \\
\hline 0.6032 & 0.968194 & 1422.94 & -0.532 & -39.66 & -0.289 \\
\hline 0.7048 & 0.988798 & 1452.99 & -0.467 & -39.85 & -0.242 \\
\hline 0.8057 & 1.007981 & 147940 & -0.347 & -36.69 & -0.088 \\
\hline 0.9034 & 1.025682 & 1503.72 & -0.213 & -26.97 & -0.212 \\
\hline \multirow{2}{*}{\multicolumn{6}{|c|}{$T=303.15 \mathrm{~K}$}} \\
\hline & & & & & \\
\hline 0.0000 & 0.795697 & 1171.68 & 0.000 & 0.00 & 0.000 \\
\hline 0.1034 & 0.83057 & 1207.83 & -0.195 & -23.38 & -0.055 \\
\hline 0.2049 & 0.861618 & 1243.3 & -0.389 & -35.51 & -0.638 \\
\hline 0.3051 & 0.890618 & 1281.84 & -0.500 & -41.29 & -0.361 \\
\hline 0.4062 & 0.917272 & 1326.76 & -0.560 & -43.30 & -0.479 \\
\hline 0.5042 & 0.94176 & 1380.88 & -0.595 & -44.49 & -0.488 \\
\hline 0.6032 & 0.964239 & 1448.99 & -0.551 & -44.03 & -0.343 \\
\hline 0.7048 & 0.98486 & 1539.41 & -0.475 & -42.77 & -0.129 \\
\hline 0.8057 & 1.004066 & 1661.34 & -0.355 & -41.06 & -0.108 \\
\hline 0.9034 & 1.021794 & 1792.34 & -0.220 & -28.80 & -0.170 \\
\hline 1.0000 & 1.03743 & 1902.17 & 0.000 & 0.00 & 0.000 \\
\hline \multicolumn{6}{|c|}{$T=308.15 \mathrm{~K}$} \\
\hline 0.0000 & 0.791626 & 1172.04 & 0.000 & 0.00 & 0.000 \\
\hline 0.1034 & 0.826496 & 1216.78 & -0.204 & -25.61 & -0.092 \\
\hline 0.2049 & 0.857562 & 1256.1 & -0.401 & -39.39 & -0.419 \\
\hline 0.3051 & 0.886574 & 1293.98 & -0.517 & -45.87 & -0.309 \\
\hline 0.4062 & 0.913243 & 1328.95 & -0.583 & -48.39 & -0.500 \\
\hline 0.5042 & 0.937751 & 1361.92 & -0.614 & -49.49 & -0.499 \\
\hline 0.6032 & 0.960249 & 1392.53 & -0.565 & -48.71 & -0.398 \\
\hline 0.7048 & 0.980896 & 1420.82 & -0.483 & -46.89 & -0.150 \\
\hline 0.8057 & 1.000129 & 1447.37 & -0.354 & -43.48 & -0.121 \\
\hline 0.9034 & 1.017885 & 1472.07 & -0.227 & -30.61 & -0.122 \\
\hline 1.0000 & 1.03355 & 1493.7 & 0.000 & 0.00 & 0.000 \\
\hline
\end{tabular}

Table 3. Continued.

\begin{tabular}{|c|c|c|c|c|c|}
\hline$x_{1}$ & $\rho / \mathrm{g}^{*} \mathrm{~cm}^{-3}$ & $u / m^{*} s^{-1}$ & $V_{m}^{E} / \mathrm{cm}^{3^{*}} \mathrm{~mol}^{-1}$ & $\Delta \kappa_{S} / \mathrm{TPa}^{-1}$ & $10^{4} \cdot \alpha_{P}^{E} / K^{-1}$ \\
\hline \multicolumn{6}{|c|}{$T=313.15 \mathrm{~K}$} \\
\hline 0.0000 & 0.787518 & 1155.16 & 0.000 & 0.00 & 0.000 \\
\hline 0.1034 & 0.822387 & 1200.05 & -0.210 & -28.44 & -0.131 \\
\hline 0.2049 & 0.85347 & 1239.52 & -0.414 & -44.00 & -0.202 \\
\hline 0.3051 & 0.882496 & 1277.55 & -0.534 & -51.36 & -0.255 \\
\hline 0.4062 & 0.909183 & 1312.59 & -0.607 & -54.21 & -0.519 \\
\hline 0.5042 & 0.93372 & 1345.68 & -0.634 & -55.29 & -0.501 \\
\hline 0.6032 & 0.956238 & 1376.43 & -0.583 & -54.01 & -0.449 \\
\hline 0.7048 & 0.97691 & 1404.84 & -0.495 & -51.55 & -0.100 \\
\hline 0.8057 & 0.996174 & 1431.58 & -0.367 & -46.83 & -0.141 \\
\hline 0.9034 & 1.013963 & 1456.39 & -0.231 & -32.14 & -0.072 \\
\hline 1.0000 & 1.02966 & 1478.25 & 0.000 & 0.00 & 0.000 \\
\hline \multicolumn{6}{|c|}{$T=318.15 \mathrm{~K}$} \\
\hline 0.0000 & 0.783359 & 1138.34 & 0.000 & 0.00 & 0.000 \\
\hline 0.1034 & 0.818231 & 1183.4 & -0.216 & -31.41 & -0.173 \\
\hline 0.2049 & 0.849338 & 1223.04 & -0.430 & -48.41 & 0.011 \\
\hline 0.3051 & 0.878383 & 1261.2 & -0.545 & -56.77 & -0.202 \\
\hline 0.4062 & 0.905093 & 1296.33 & -0.633 & -60.07 & -0.546 \\
\hline 0.5042 & 0.929649 & 1329.59 & -0.663 & -60.88 & -0.513 \\
\hline 0.6032 & 0.952199 & 1360.45 & -0.608 & -59.38 & -0.501 \\
\hline 0.7048 & 0.9729 & 1388.99 & -0.495 & -56.16 & -0.051 \\
\hline 0.8057 & 0.992199 & 1415.93 & -0.371 & -50.39 & -0.155 \\
\hline 0.9034 & 1.01002 & 1440.85 & -0.234 & -34.00 & -0.032 \\
\hline 1.0000 & 1.02575 & 1462.82 & 0.000 & 0.00 & 0.000 \\
\hline
\end{tabular}


Table 4. Densities, $\rho$, speeds of sound, $u$, excess molar volumes, $V_{m}^{E}$, deviation in isentropic compressibility, $\Delta \kappa_{s}$, and excess thermal expansion coefficients $\alpha_{P}^{E}$ as functions of mole fraction, $x_{1}$ of glycerol for $\{$ benzyl alcohol (1) + 2-propanol (2)\} mixtures at the temperatures (298.15 to 318.15) K.

\begin{tabular}{|c|c|c|c|c|c|}
\hline$x_{1}$ & $\rho / \mathrm{g}^{*} \mathrm{~cm}^{-3}$ & $u / m^{*} s^{-1}$ & $V_{m}^{E} / \mathrm{cm}^{3 *} \mathrm{~mol}^{-1}$ & $\Delta \kappa_{S} / \mathrm{TPa}^{-1}$ & $10^{4} \cdot \alpha_{P}^{E} / K^{-1}$ \\
\hline \multicolumn{6}{|c|}{$T=298.15 \mathrm{~K}$} \\
\hline 0.0000 & 0.781279 & 1139.36 & 0.000 & 0.00 & 0.000 \\
\hline 0.1034 & 0.817946 & 1193.67 & -0.099 & -17.51 & -0.228 \\
\hline 0.2049 & 0.852029 & 1243.89 & -0.265 & -29.80 & -0.435 \\
\hline 0.3051 & 0.882397 & 1290.16 & -0.398 & -36.69 & -0.155 \\
\hline 0.4062 & 0.911462 & 1332.39 & -0.522 & -41.01 & -0.676 \\
\hline 0.5042 & 0.937684 & 1371.74 & -0.551 & -42.49 & -0.680 \\
\hline 0.6032 & 0.961894 & 1408.05 & -0.589 & -43.38 & -0.751 \\
\hline 0.7048 & 0.98388 & 1440.92 & -0.533 & -42.35 & -0.771 \\
\hline 0.8057 & 1.005465 & 1473.06 & -0.483 & -38.09 & -0.455 \\
\hline 0.9034 & 1.024805 & 1501.16 & -0.302 & -25.71 & -0.180 \\
\hline \multirow{2}{*}{\multicolumn{6}{|c|}{$T=303.15 \mathrm{~K}$}} \\
\hline & & & & & \\
\hline 0.0000 & 0.777005 & 1121.96 & 0.000 & 0.00 & 0.000 \\
\hline 0.1034 & 0.813696 & 1176.35 & -0.112 & -19.83 & -0.298 \\
\hline 0.2049 & 0.847815 & 1226.98 & -0.288 & -33.88 & -0.504 \\
\hline 0.3051 & 0.877727 & 1273.37 & -0.418 & -41.29 & -0.471 \\
\hline 0.4062 & 0.907328 & 1315.64 & -0.542 & -46.01 & -0.711 \\
\hline 0.5042 & 0.933566 & 1355.17 & -0.584 & -47.48 & -0.706 \\
\hline 0.6032 & 0.957838 & 1391.61 & -0.621 & -47.86 & -0.725 \\
\hline 0.7048 & 0.979869 & 1424.67 & -0.569 & -46.29 & -0.601 \\
\hline 0.8057 & 1.001501 & 1456.9 & -0.501 & -41.08 & -0.339 \\
\hline 0.9034 & 1.020897 & 1485.53 & -0.310 & -27.03 & -0.058 \\
\hline 1.0000 & 1.03743 & 1509.43 & 0.000 & 0.00 & 0.000 \\
\hline \multicolumn{6}{|c|}{$T=308.15 \mathrm{~K}$} \\
\hline 0.0000 & 0.77265 & 1104.33 & 0.000 & 0.00 & 0.000 \\
\hline 0.1034 & 0.809377 & 1158.99 & -0.127 & -22.61 & -0.355 \\
\hline 0.2049 & 0.843536 & 1209.79 & -0.314 & -38.39 & -0.588 \\
\hline 0.3051 & 0.872984 & 1256.37 & -0.437 & -46.49 & -0.801 \\
\hline 0.4062 & 0.90314 & 1298.78 & -0.587 & -51.70 & -0.742 \\
\hline 0.5042 & 0.929446 & 1338.45 & -0.615 & -53.08 & -0.731 \\
\hline 0.6032 & 0.953748 & 1375.1 & -0.653 & -53.05 & -0.719 \\
\hline 0.7048 & 0.975833 & 1408.33 & -0.583 & -50.16 & -0.441 \\
\hline 0.8057 & 0.997516 & 1440.79 & -0.512 & -44.18 & -0.220 \\
\hline 0.9034 & 1.016965 & 1469.59 & -0.308 & -28.89 & 0.065 \\
\hline 1.0000 & 1.03355 & 1493.7 & 0.000 & 0.00 & 0.000 \\
\hline
\end{tabular}

Table 4. continued.

\begin{tabular}{|c|c|c|c|c|c|}
\hline$x_{1}$ & $\rho / \mathrm{g}^{*} \mathrm{~cm}^{-3}$ & $u / m^{*} \mathrm{~s}^{-1}$ & $V_{m}^{E} / \mathrm{cm}^{3^{*}} \mathrm{~mol}^{-1}$ & $\Delta \kappa_{S} / \mathrm{TPa}^{-1}$ & $10^{4} \cdot \alpha_{P}^{E} / K^{-1}$ \\
\hline \multicolumn{6}{|c|}{$T=313.15 \mathrm{~K}$} \\
\hline 0.0000 & 0.768208 & 1086.63 & 0.000 & 0.00 & 0.000 \\
\hline 0.1034 & 0.804985 & 1141.59 & -0.146 & -25.72 & -0.422 \\
\hline 0.2049 & 0.839199 & 1192.6 & -0.343 & -43.38 & -0.668 \\
\hline 0.3051 & 0.856654 & 1238.99 & -0.494 & -52.55 & -1.122 \\
\hline 0.4062 & 0.898909 & 1281.95 & -0.622 & -58.01 & -0.781 \\
\hline 0.5042 & 0.925273 & 1321.81 & -0.648 & -59.07 & -0.761 \\
\hline 0.6032 & 0.949619 & 1358.65 & -0.685 & -58.71 & -0.700 \\
\hline 0.7048 & 0.97177 & 1392.08 & -0.603 & -55.00 & -0.272 \\
\hline 0.8057 & 0.993509 & 1424.77 & -0.520 & -47.63 & -0.101 \\
\hline 0.9034 & 1.013014 & 1453.78 & -0.304 & -30.88 & 0.191 \\
\hline 1.0000 & 1.02966 & 1478.25 & 0.000 & 0.00 & 0.000 \\
\hline \multicolumn{6}{|c|}{$T=318.15 \mathrm{~K}$} \\
\hline 0.0000 & 0.76367 & 1086.84 & 0.000 & 0.00 & 0.000 \\
\hline 0.1034 & 0.800051 & 1124.13 & -0.168 & -29.23 & -0.490 \\
\hline 0.2049 & 0.834793 & 1175.37 & -0.377 & -48.40 & -0.745 \\
\hline 0.3051 & 0.848137 & 1221.97 & -0.545 & -59.22 & -1.459 \\
\hline 0.4062 & 0.894635 & 1265.15 & -0.654 & -64.83 & -0.822 \\
\hline 0.5042 & 0.921059 & 1305.24 & -0.685 & -65.59 & -0.791 \\
\hline 0.6032 & 0.945482 & 1342.26 & -0.716 & -64.86 & -0.683 \\
\hline 0.7048 & 0.967664 & 1375.9 & -0.613 & -60.13 & -0.102 \\
\hline 0.8057 & 0.989477 & 1408.88 & -0.524 & -51.51 & 0.012 \\
\hline 0.9034 & 1.009043 & 1438.08 & -0.297 & -32.91 & 0.318 \\
\hline 1.0000 & 1.02575 & 1462.82 & 0.000 & 0.00 & 0.000 \\
\hline
\end{tabular}


Table 5. Densities, $\rho$, speeds of sound, $u$, excess molar volumes, $V_{m}^{E}$, deviation in isentropic compressibility, $\Delta \kappa_{s}$, and excess thermal expansion coefficients $\alpha_{P}^{E}$ as functions of mole fraction, $x_{1}$ of glycerol for \{\{benzyl alcohol (1) +1,3-propanol (2)\} mixtures at the temperatures (298.15 to 318.15) K.

\begin{tabular}{|c|c|c|c|c|c|}
\hline$x_{1}$ & $\rho / \mathrm{g}^{*} \mathrm{~cm}^{-3}$ & $u / \mathrm{m}^{*} \mathrm{~s}^{-1}$ & $V_{m}^{E} / \mathrm{cm}^{3^{*}} \mathrm{~mol}^{-1}$ & $\Delta \kappa_{S} / \mathrm{TPa}^{-1}$ & $10^{4} \cdot \alpha_{P}^{E} / K^{-1}$ \\
\hline \multicolumn{6}{|c|}{$T=298.15 \mathrm{~K}$} \\
\hline 0.0000 & 1.04129 & 1525.13 & 0.000 & 0.00 & 0.000 \\
\hline 0.1043 & 1.05728 & 1543.09 & 0.041 & 4.72 & 0.052 \\
\hline 0.2038 & 1.07409 & 1562.03 & 0.027 & 8.61 & 0.111 \\
\hline 0.3053 & 1.09245 & 1584.55 & 0.004 & 11.50 & 0.113 \\
\hline 0.4087 & 1.11251 & 1612.10 & -0.024 & 13.02 & 0.120 \\
\hline 0.5045 & 1.13247 & 1642.75 & -0.056 & 13.12 & 0.184 \\
\hline 0.6037 & 1.15432 & 1680.15 & -0.068 & 12.25 & 0.101 \\
\hline 0.7059 & 1.17848 & 1727.55 & -0.078 & 9.71 & 0.072 \\
\hline 0.8039 & 1.20330 & 1783.84 & -0.077 & 5.79 & 0.030 \\
\hline 0.9069 & 1.23127 & 1851.74 & -0.063 & 1.63 & 0.059 \\
\hline \multirow{2}{*}{\multicolumn{6}{|c|}{$T=303.15 \mathrm{~K}$}} \\
\hline & & & & & \\
\hline 0.0000 & 1.03743 & 1509.43 & 0.000 & 0.00 & 0.000 \\
\hline 0.1043 & 1.05343 & 1527.76 & 0.044 & 4.73 & 0.061 \\
\hline 0.2038 & 1.07025 & 1547.04 & 0.032 & 8.62 & 0.111 \\
\hline 0.3053 & 1.08865 & 1570.02 & 0.010 & 11.47 & 0.120 \\
\hline 0.4087 & 1.10876 & 1597.69 & -0.019 & 13.11 & 0.123 \\
\hline 0.5045 & 1.12876 & 1628.77 & -0.050 & 13.16 & 0.182 \\
\hline 0.6037 & 1.15071 & 1666.50 & -0.065 & 12.22 & 0.108 \\
\hline 0.7059 & 1.17497 & 1714.34 & -0.076 & 9.64 & 0.101 \\
\hline 0.8039 & 1.19991 & 1770.93 & -0.078 & 5.62 & 0.090 \\
\hline 0.9069 & 1.22798 & 1839.23 & -0.062 & 1.52 & 0.105 \\
\hline 1.0000 & 1.25461 & 1902.17 & 0.000 & 0.00 & 0.000 \\
\hline \multicolumn{6}{|c|}{$T=308.15 \mathrm{~K}$} \\
\hline 0.0000 & 1.03355 & 1493.70 & 0.000 & 0.00 & 0.000 \\
\hline 0.1043 & 1.04955 & 1512.33 & 0.048 & 4.81 & 0.069 \\
\hline 0.2038 & 1.06639 & 1531.86 & 0.038 & 8.73 & 0.108 \\
\hline 0.3053 & 1.08483 & 1555.20 & 0.016 & 11.58 & 0.121 \\
\hline 0.4087 & 1.10499 & 1583.27 & -0.012 & 13.20 & 0.122 \\
\hline 0.5045 & 1.12501 & 1614.62 & -0.040 & 13.22 & 0.181 \\
\hline 0.6037 & 1.14708 & 1652.86 & -0.060 & 12.26 & 0.120 \\
\hline 0.7059 & 1.17141 & 1701.05 & -0.070 & 9.63 & 0.132 \\
\hline 0.8039 & 1.19644 & 1758.22 & -0.071 & 5.51 & 0.150 \\
\hline 0.9069 & 1.22462 & 1827.15 & -0.055 & 1.42 & 0.163 \\
\hline 1.0000 & 1.25150 & 1890.42 & 0.000 & 0.00 & 0.000 \\
\hline
\end{tabular}

Table 5. Continued.

\begin{tabular}{|c|c|c|c|c|c|}
\hline$x_{1}$ & $\rho / \mathrm{g}^{*} \mathrm{~cm}^{-3}$ & $u / \mathrm{m}^{*} \mathrm{~s}^{-1}$ & $V_{m}^{E} / \mathrm{cm}^{3^{*}} \mathrm{~mol}^{-1}$ & $\Delta \kappa_{S} / \mathrm{TPa}^{-1}$ & $10^{4} \cdot \alpha_{P}^{E} / K^{-1}$ \\
\hline \multicolumn{6}{|c|}{$T=313.15 \mathrm{~K}$} \\
\hline 0.0000 & 1.02966 & 1478.25 & 0.000 & 0.00 & 0.000 \\
\hline 0.1043 & 1.04566 & 1497.01 & 0.052 & 4.95 & 0.075 \\
\hline 0.2038 & 1.06252 & 1516.79 & 0.044 & 8.93 & 0.102 \\
\hline 0.3053 & 1.08100 & 1540.52 & 0.022 & 11.74 & 0.128 \\
\hline 0.4087 & 1.10122 & 1568.94 & -0.006 & 13.34 & 0.130 \\
\hline 0.5045 & 1.12125 & 1600.59 & -0.031 & 13.43 & 0.178 \\
\hline 0.6037 & 1.14343 & 1639.24 & -0.053 & 12.36 & 0.131 \\
\hline 0.7059 & 1.16784 & 1687.96 & -0.063 & 9.65 & 0.163 \\
\hline 0.8039 & 1.19294 & 1745.76 & -0.063 & 5.50 & 0.210 \\
\hline 0.9069 & 1.22123 & 1815.05 & -0.046 & 1.42 & 0.218 \\
\hline 1.0000 & 1.24838 & 1879.08 & 0.000 & 0.00 & 0.000 \\
\hline \multicolumn{6}{|c|}{$T=318.15 \mathrm{~K}$} \\
\hline 0.0000 & 1.02575 & 1462.82 & 0.000 & 0.00 & 0.000 \\
\hline 0.1043 & 1.04175 & 1481.8 & 0.056 & 5.08 & 0.081 \\
\hline 0.2038 & 1.05864 & 1501.85 & 0.049 & 9.12 & 0.100 \\
\hline 0.3053 & 1.07715 & 1525.86 & 0.028 & 11.92 & 0.132 \\
\hline 0.4087 & 1.09742 & 1554.65 & 0.000 & 13.50 & 0.131 \\
\hline 0.5045 & 1.11751 & 1586.66 & -0.025 & 13.53 & 0.173 \\
\hline 0.6037 & 1.13977 & 1625.72 & -0.049 & 12.41 & 0.142 \\
\hline 0.7059 & 1.16424 & 1674.9 & -0.057 & 9.69 & 0.193 \\
\hline 0.8039 & 1.18941 & 1733.17 & -0.055 & 5.49 & 0.271 \\
\hline 0.9069 & 1.21782 & 1803.06 & -0.039 & 1.38 & 0.272 \\
\hline 1.0000 & 1.24521 & 1867.86 & 0.000 & 0.00 & 0.000 \\
\hline
\end{tabular}


Table 6. Densities, $\rho$, speeds of sound, $u$, excess molar volumes, $V_{m}^{E}$, deviation in isentropic compressibilities, $\Delta \kappa_{s}$, and excess thermal expansion coefficients $\alpha_{P}^{E}$ as functions of mole fraction, $x_{1}$ of glycerol for \{\{benzilalcohol (1) + phenylethanol (2)\} mixtures at the temperatures (298.15 to 318.15) K.

\begin{tabular}{|c|c|c|c|c|c|}
\hline$x_{1}$ & $\rho / g^{*} \mathrm{~cm}^{-3}$ & $u / m^{*} s^{-1}$ & $V_{m}^{E} / \mathrm{cm}^{3^{*}} \mathrm{~mol}^{-1}$ & $\Delta \kappa_{S} / \mathrm{TPa}^{-1}$ & $10^{4} \cdot \alpha_{P}^{E} / \mathrm{K}^{-1}$ \\
\hline \multicolumn{6}{|c|}{$T=298.15 \mathrm{~K}$} \\
\hline 0.0000 & 1.04129 & 1525.13 & 0.000 & 0.00 & 0.000 \\
\hline 0.1043 & 1.05728 & 1543.09 & 0.041 & 4.77 & 0.057 \\
\hline 0.2038 & 1.07409 & 1562.03 & 0.027 & 8.63 & 0.115 \\
\hline 0.3053 & 1.09245 & 1584.55 & 0.004 & 11.50 & 0.116 \\
\hline 0.4087 & 1.11251 & 1612.10 & -0.024 & 13.01 & 0.122 \\
\hline 0.5045 & 1.13247 & 1642.75 & -0.056 & 13.19 & 0.189 \\
\hline 0.6037 & 1.15432 & 1680.15 & -0.068 & 12.31 & 0.101 \\
\hline 0.8039 & 1.20330 & 1783.84 & -0.077 & 5.81 & 0.033 \\
\hline 0.9069 & 1.23127 & 1851.74 & -0.063 & 1.66 & 0.061 \\
\hline 1.0000 & 1.25777 & 1914.44 & 0.000 & 0.00 & 0.000 \\
\hline \multicolumn{6}{|c|}{$T=303.15 \mathrm{~K}$} \\
\hline 0.0000 & 1.03743 & 1509.43 & 0.000 & 0.00 & 0.000 \\
\hline 0.1043 & 1.05343 & 1527.76 & 0.044 & 4.76 & 0.065 \\
\hline 0.2038 & 1.07025 & 1547.04 & 0.032 & 8.63 & 0.113 \\
\hline 0.3053 & 1.08865 & 1570.02 & 0.010 & 11.45 & 0.122 \\
\hline 0.5045 & 1.12876 & 1628.77 & -0.050 & 13.18 & 0.183 \\
\hline 0.6037 & 1.15071 & 1666.50 & -0.065 & 12.26 & 0.111 \\
\hline 0.7059 & 1.17497 & 1714.34 & -0.076 & 9.66 & 0.107 \\
\hline 0.8039 & 1.19991 & 1770.93 & -0.078 & 5.65 & 0.092 \\
\hline 0.9069 & 1.22798 & 1839.23 & -0.062 & 1.56 & 0.113 \\
\hline 1.0000 & 1.25461 & 1902.17 & 0.000 & 0.00 & 0.000 \\
\hline \multicolumn{6}{|c|}{$T=308.15 \mathrm{~K}$} \\
\hline 0.0000 & 1.03355 & 1493.70 & 0.000 & 0.00 & 0.000 \\
\hline 0.1043 & 1.04955 & 1512.33 & 0.048 & 4.84 & 0.070 \\
\hline 0.2038 & 1.06639 & 1531.86 & 0.038 & 8.73 & 0.110 \\
\hline 0.3053 & 1.08483 & 1555.20 & 0.016 & 11.60 & 0.126 \\
\hline 0.4087 & 1.10499 & 1583.27 & -0.012 & 13.20 & 0.125 \\
\hline 0.5045 & 1.12501 & 1614.62 & -0.040 & 13.28 & 0.183 \\
\hline 0.6037 & 1.14708 & 1652.86 & -0.060 & 12.27 & 0.122 \\
\hline 0.7059 & 1.17141 & 1701.05 & -0.070 & 9.61 & 0.133 \\
\hline 0.8039 & 1.19644 & 1758.22 & -0.071 & 5.59 & 0.153 \\
\hline 0.9069 & 1.22462 & 1827.15 & -0.055 & 1.45 & 0.167 \\
\hline
\end{tabular}

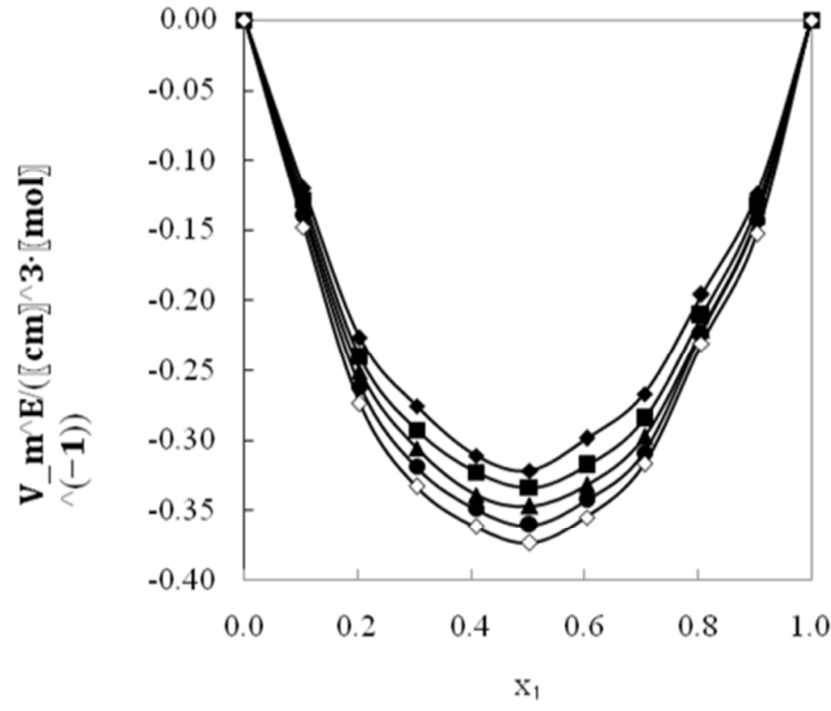

Figure 1. Plot of: (a) excess molar volumes against mole fraction for $\{$ benzylalcohol (1) + 1,2-propandiol (2) $\}$ at the temperatures $298.15 \mathrm{~K}$; $303.15 \mathrm{~K} ; \boldsymbol{\boldsymbol { \sigma }}, 308.15 \mathrm{~K} ; \boldsymbol{\Delta}, 313.15 \mathrm{~K} ; \boldsymbol{\bullet}, 318.15 \mathrm{~K}$; $\diamond$.

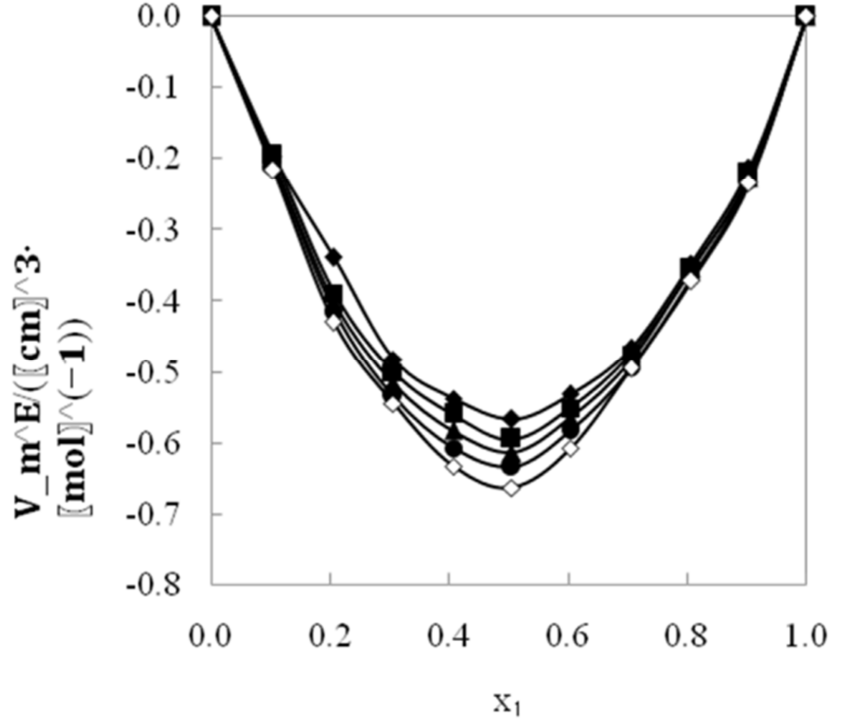

Figure 2. Plot of: (a) excess molar volumes against mole fraction for \{benzylalcohol (1) + 1-propanol (2)\} at the temperatures $298.15 \mathrm{~K}$; $303.15 \mathrm{~K} ; \boldsymbol{\boldsymbol { \sigma }}, 308.15 \mathrm{~K} ; \boldsymbol{\Delta}, 313.15 \mathrm{~K} ; \boldsymbol{\bullet}, 318.15 \mathrm{~K}$; $\diamond$. 


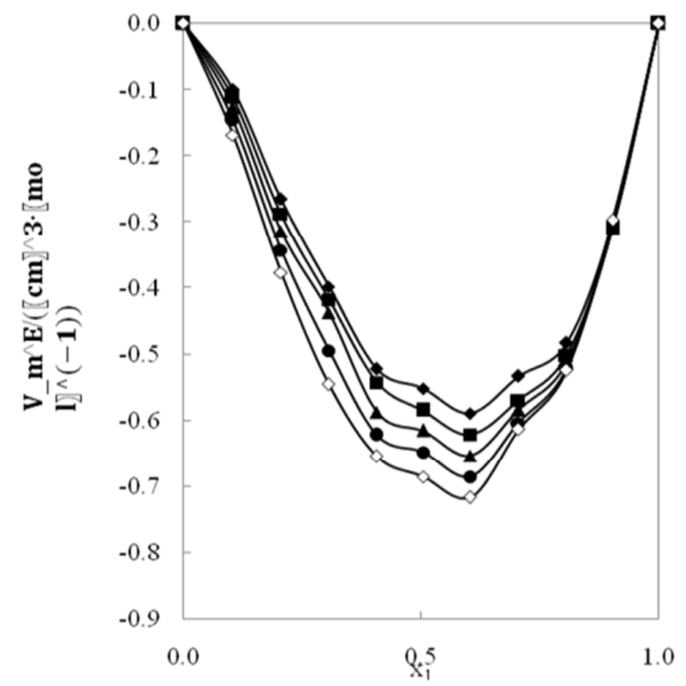

Figure 3. Plot of: (a) excess molar volumes against mole fraction for $\{$ benzylalcohol (1) + 2-propanol (2)\} at the temperatures $298.15 \mathrm{~K}$; $303.15 \mathrm{~K} ; \boldsymbol{\boldsymbol { \sigma }}, 308.15 \mathrm{~K} ; \boldsymbol{\Delta}, 313.15 \mathrm{~K} ; \boldsymbol{\bullet}, 318.15 \mathrm{~K}$; $\diamond$.

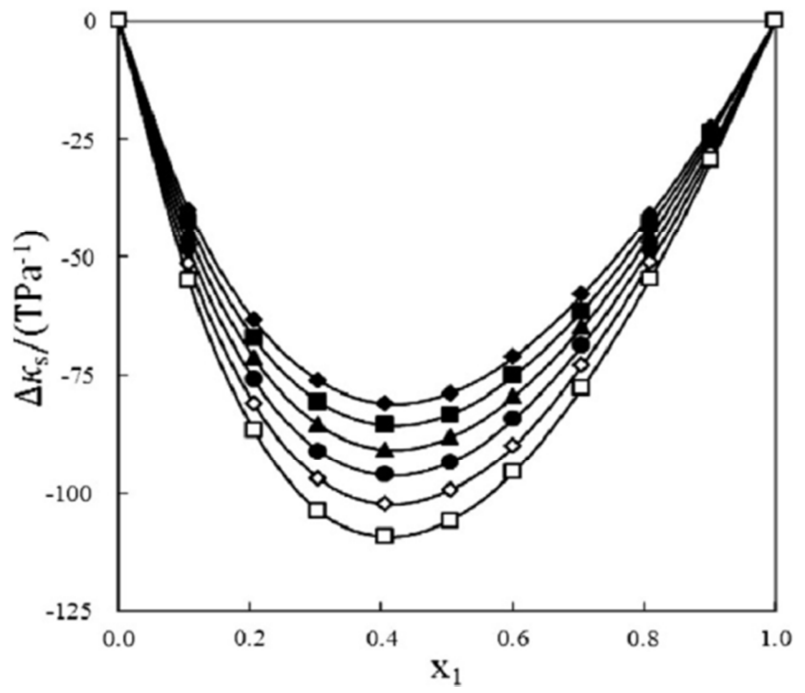

Figure 4. Plot of: (a) excess molar volumes against mole fraction for \{ benzylalcohol (1) + 1,3-propandiol (2) \} at the temperatures $298.15 \mathrm{~K}$; $303.15 \mathrm{~K} ; \boldsymbol{\boldsymbol { }}, 308.15 \mathrm{~K} ; \boldsymbol{\Delta}, 313.15 \mathrm{~K} ; \boldsymbol{\bullet}, 318.15 \mathrm{~K}$; $\diamond$.

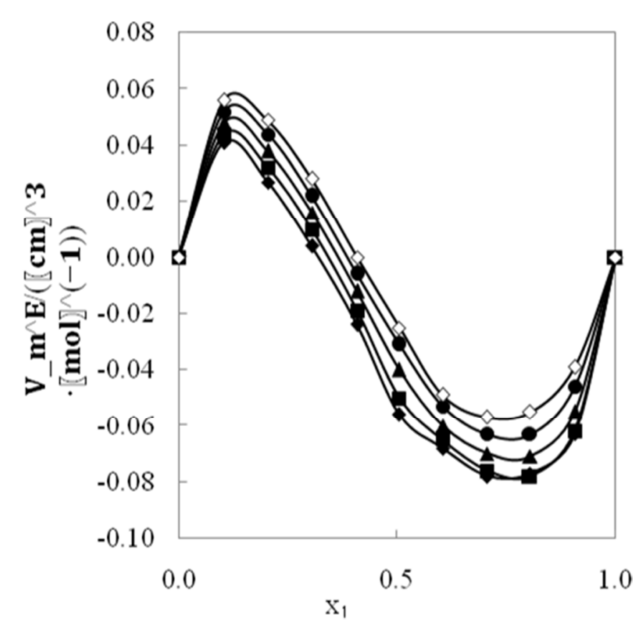

Figure 5. Plot of: (a) excess molar volumes, against mole fraction for \{benzylalcohol (1) + phenylethanol (2)\} at the temperatures $298.15 \mathrm{~K}$; $303.15 \mathrm{~K} ; \boldsymbol{\boldsymbol { \sigma }}, 308.15 \mathrm{~K} ; \boldsymbol{\Delta}, 313.15 \mathrm{~K} ; \boldsymbol{\bullet}, 318.15 \mathrm{~K} ; \diamond$.

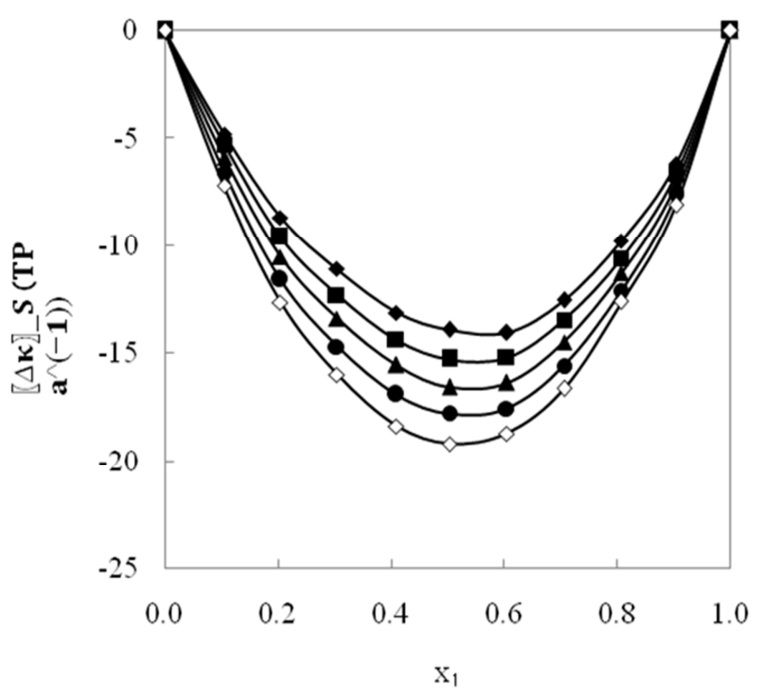

Figure 6. Plot of (b) deviations in isentropic compressibility against mole fraction for \{benzylalcohol (1) + 1,2-propandiol (2)\} at the temperatures

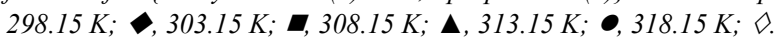

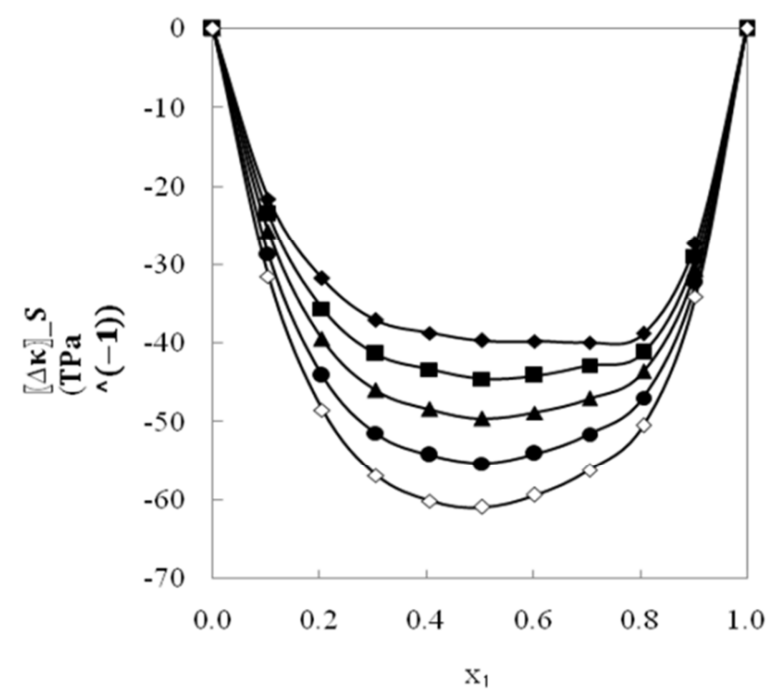

Figure 7. Plot of: (b) deviations in isentropic compressibility against mole fraction for \{benzylalcohol (1) + 1-propanol (2)\} at the temperatures 298.15 $\mathrm{K} ; \diamond, 303.15 \mathrm{~K} ; \boldsymbol{\boldsymbol { \Delta }}, 308.15 \mathrm{~K} ; \boldsymbol{\Delta}, 313.15 \mathrm{~K} ; \boldsymbol{\bullet}, 318.15 \mathrm{~K} ; \diamond$.

\subsection{Speed of Sound Excess Isentropic Compressibility}

By employing the experimental values of $\rho$ and $u$, deviations in isentropic compressibility, $\Delta \kappa_{s}$ were obtained from the equation:

$$
\Delta \kappa_{\mathrm{s}}=\kappa_{\mathrm{s}}-\sum_{\mathrm{i}=1} \mathrm{X}_{\mathrm{i}} \kappa_{\mathrm{s}, \mathrm{i}}
$$

Where $\kappa_{\mathrm{s}, \mathrm{I}}$ and $\kappa_{\mathrm{s}}$ are the isentropic compressibilities of the pure component $i$ and mixtures, respectively. The isentropic compressibility, $\kappa_{\mathrm{s}}$, was calculated using the Newton-Laplace equation:

$$
\kappa_{\mathrm{s}}=1 / \rho \mathrm{u}^{2}
$$




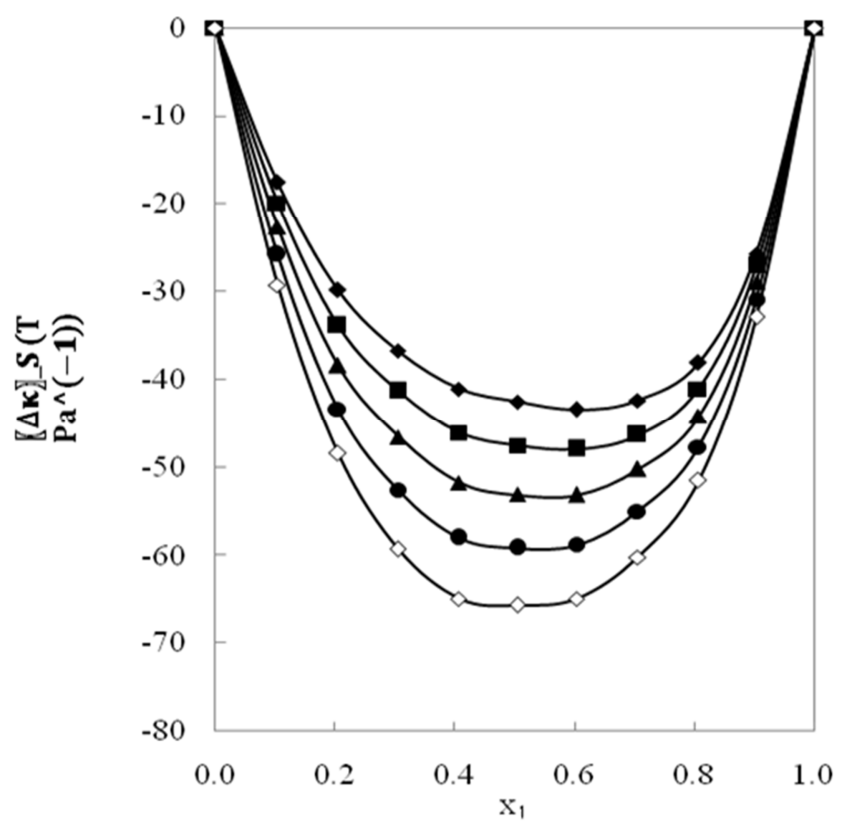

Figure 8. Plot of: (b) deviations in isentropic compressibility against mole fraction for \{benzylalcohol (1) + 2-propanol (2)\} at the temperatures 298.15

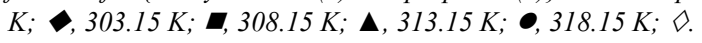

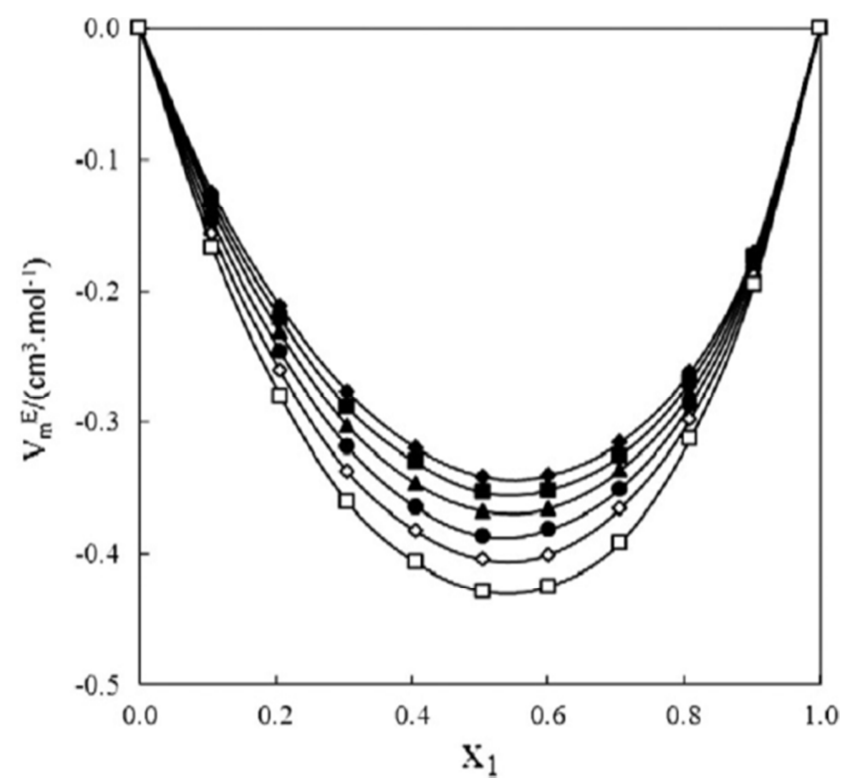

Figure 9. Plot of: (b) deviations in isentropic compressibility against mole fraction for \{ benzylalcohol (1) + 1,3-propandiol (2) \} at the temperatures $298.15 \mathrm{~K} ; \boldsymbol{\diamond}, 303.15 \mathrm{~K} ; \boldsymbol{\bullet}, 308.15 \mathrm{~K} ; \boldsymbol{\Lambda}, 313.15 \mathrm{~K} ; \boldsymbol{\bullet}, 318.15 \mathrm{~K} ; \diamond$.

The calculated $\Delta \kappa s$ values for the selected binary mixtures are listed in Tables (2) - (6). The change of this property has been shown in Figures (1-5) (b). It is evident from Figure 1-5) (b) and Tables (2) - (6) that the $\Delta \kappa s$ values are negative over the entire mole fraction range and become more negative with increasing temperature for all binary mixtures. Thus, the mixtures are less compressible than the pure components, i.e., the greater resistance to compression (enhanced rigidity) is observed. The most of the systems show both enhanced rigidity $(\Delta \kappa s<0)$ and contraction $\left(V_{m}^{E}<0\right)$ over the entire composition range. In other words, the volume decreases (more compact packing of molecules), and simultaneously the whole system becomes more rigid (less compressible). Interpretation of the $\Delta \kappa s$ data is generally not simple because the $\Delta \kappa s$ values are affected by both the molecular packing and the patterns of molecular aggregation induced by the molecular interactions. However, in these three binary systems it seems that interpretation of negative $\Delta \kappa \mathrm{s}$ values is the same as negative $V_{m}^{E}$ values.

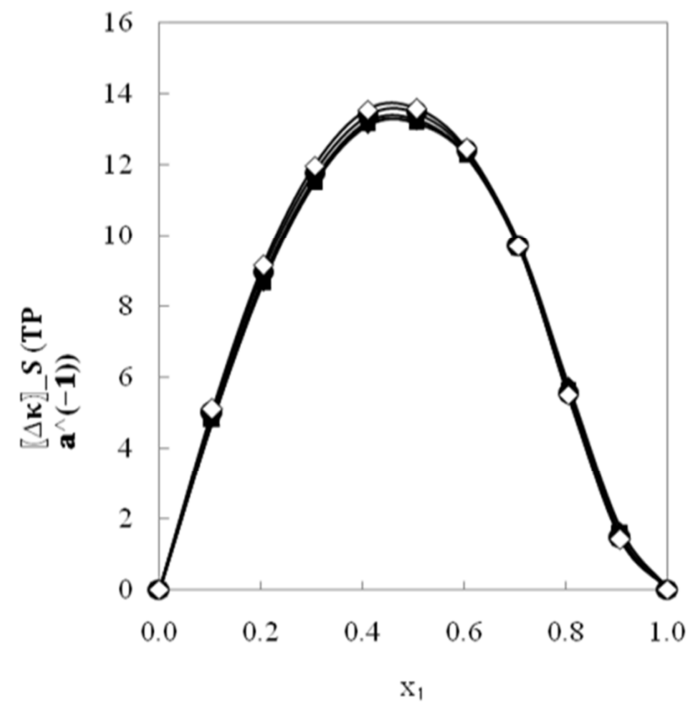

Figure 10. Plot of: (b) deviations in isentropic compressibility against mole fraction for \{benzylalcohol (1) + phenylethanol (2)\} at the temperatures

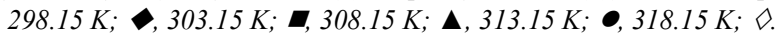

\subsection{Excess Thermal Expansion Coefficient}

The excess thermal expansion coefficient, $\alpha_{P}^{E}$, were determined using the following equation:

$$
\alpha_{P}^{E}=\alpha_{P}-\sum_{i=1}^{2} \varphi_{i} \alpha_{P, i}
$$

where $\alpha_{P}$ and $\alpha_{P, i}$ are thermal expansion coefficients of the mixture and pure component $i$, respectively; $\varphi_{i}$ represents the volume fraction of component $i$ obtained through the relation:

$$
\varphi_{i}=\frac{x_{i} V_{i}^{*}}{\sum_{i=1}^{2} x_{i} V_{i}^{*}}
$$

Where $V_{i}^{*}$ is the molar volume of the pure component $\mathrm{i}$. The thermal expansion coefficient, $\alpha_{P}$, was calculated from:

$$
\alpha_{P}=-\frac{1}{\rho}\left(\frac{\partial \rho}{\partial T}\right)_{P}
$$

The measured densities for all components were fitted with temperature by the following simple polynomial equation:

$$
\rho(T)=\sum_{i=0}^{2} a_{i}(T / K)^{i}
$$

Where $a_{i}$ refer to the fitting coefficients.

By employing Esq. (4) - (7) the numerical values of $\alpha_{P}^{E}$ were calculated for the selected binary mixtures. The numerical results have been tabulated in Tables (2) - (6). From Tables (2) - (6) and Figures (1) - (5) (c) it is obvious that the values of excess thermal expansion coefficients $\alpha_{p}^{E}$ are negative over the whole composition range for the 
considered mixtures In general, negative $\alpha_{p}^{E}$ values indicate the presence of strong interaction between the components in the mixtures.

The composition dependence of $V_{m}^{E}, \Delta \kappa_{s}$ and $\alpha_{P}^{E}$ of all binary mixtures, were fitted to a Redlich-Kister type polynomial equation:

$$
Y^{E}=x_{1} x_{2} \sum_{i=0}^{3} A_{i}\left(1-2 x_{1}\right)^{i}
$$

Where $Y^{E}$ stands for $V_{m}^{E}, \Delta \kappa_{s}$ and $\alpha_{P}^{E} . A_{i}$ are the polynomial coefficients and $x$ is the mole fraction. The coefficients $A_{i}$ in Eq. 8) were allowed to vary using a non- linear least squares method and are listed in Table (7). To ascertain the validity of the polynomial coefficients, the standard deviations $\sigma\left(Y^{E}\right)$ are also calculated from the following expression and included in Table (7).

$$
\sigma\left(Y^{E}\right)=\left[\sum_{i=1}^{n} \frac{\left(Y_{i}-Y_{i}(\text { calc. })\right)^{2}}{n-p}\right]^{1 / 2}
$$

Where $n$ is the number of experimental data and $p$ is the number of parameters.

Table 7. Coefficients, $A_{i}$ from Eq. (8) for $V_{m}^{E}, \Delta \kappa_{s}, \alpha_{P}^{E}$ and standard deviations $\sigma$ for benzilalcohol + 1,3-propandiol, 1,3-propandiol, 1-propanol, 2-propanol and t-butanol mixtures at temperatures from 298.15 to $318.15 \mathrm{~K}$.

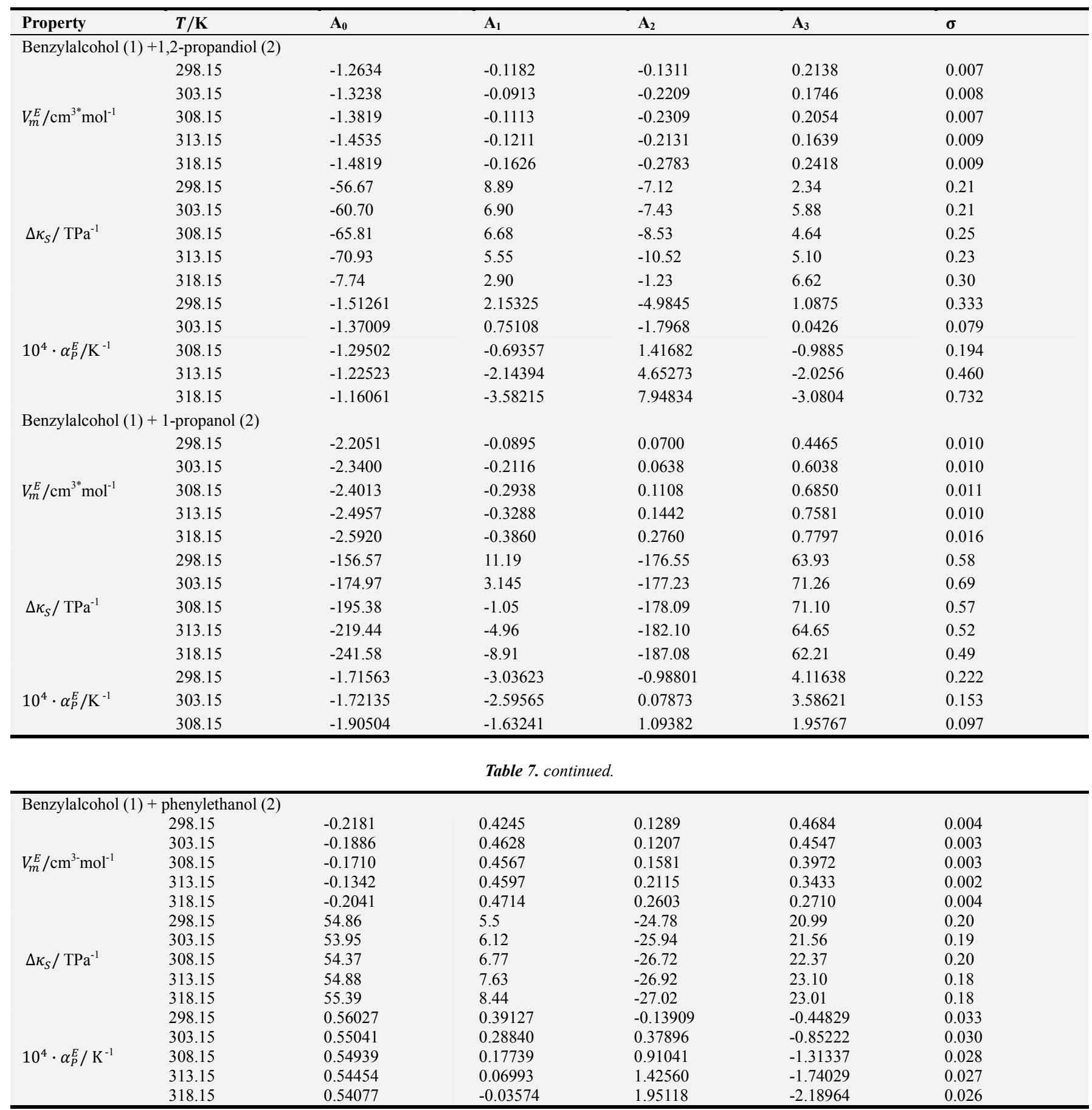




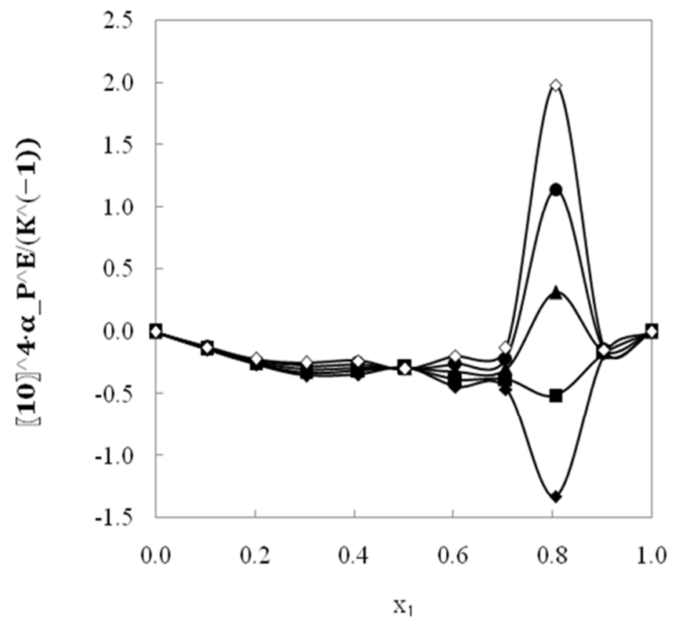

Figure 11. Plot of: (c) excess thermal expansion coefficients against mole fraction for \{benzylalcohol (1) + 1,2-propandiol (2)\} at the temperatures $298.15 \mathrm{~K} ; \bullet, 303.15 \mathrm{~K} ; \boldsymbol{\square}, 308.15 \mathrm{~K} ; \boldsymbol{\Lambda}, 313.15 \mathrm{~K} ; \bullet, 318.15 \mathrm{~K} ; \diamond$

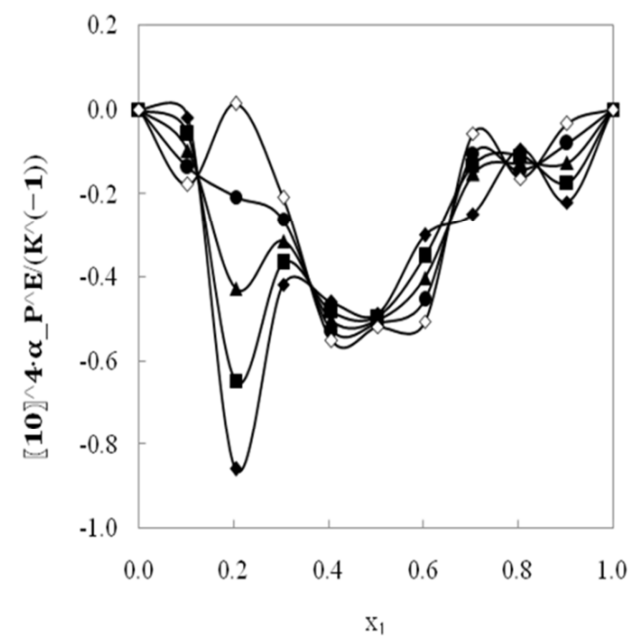

Figure 12. Plot of: (c) excess thermal expansion coefficients against mole fraction for \{benzylalcohol (1) + 1-propanol (2)\} at the temperatures 298.15 $\mathrm{K} ; \bullet, 303.15 \mathrm{~K} ; \boldsymbol{\square}, 308.15 \mathrm{~K} ; \boldsymbol{\Lambda}, 313.15 \mathrm{~K} ; \bullet, 318.15 \mathrm{~K} ; \diamond$.

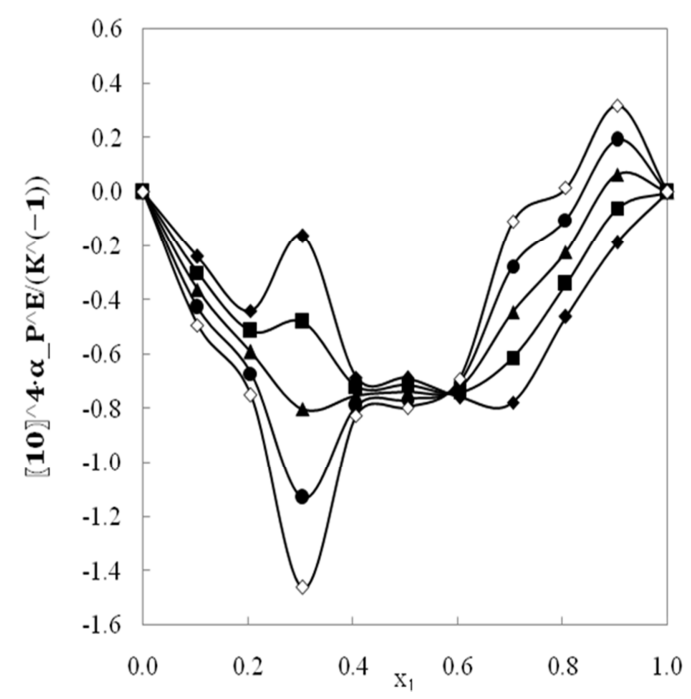

Figure 13. Plot of (c) excess thermal expansion coefficients against mole fraction for \{benzylalcohol (1) + 2-propanol (2)\} at the temperatures 298.15

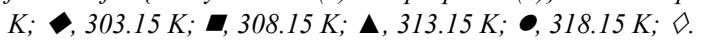

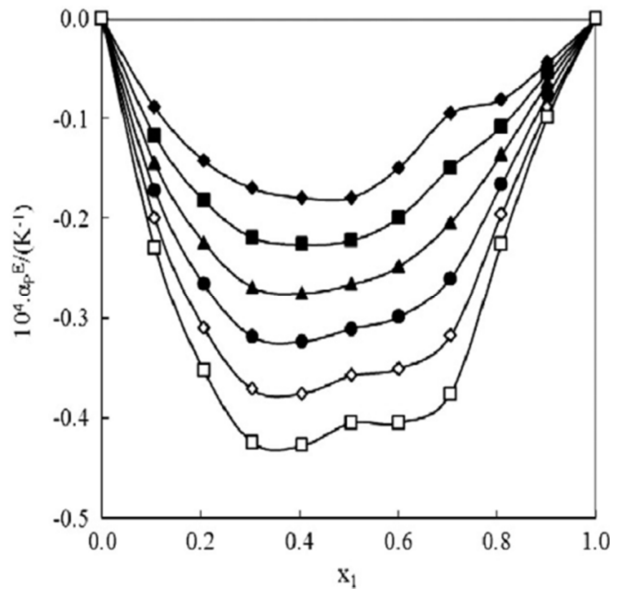

Figure 14. Plot of: (c) excess thermal expansion coefficients against mole fraction for \{benzylalcohol (1) + 1,3-propandiol (2)\} at the temperatures

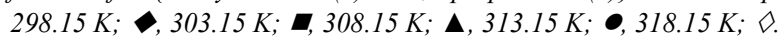

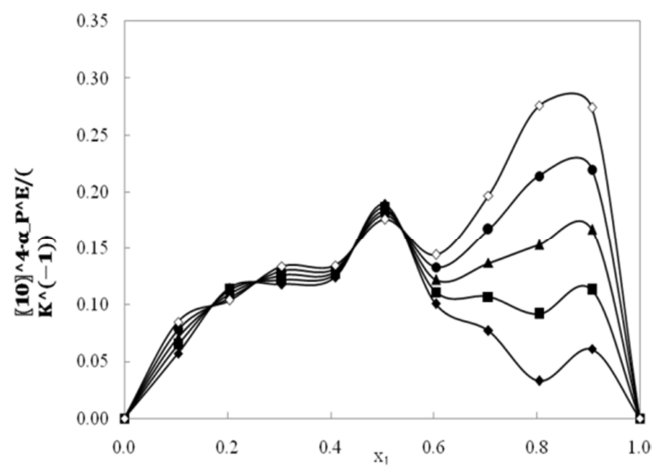

Figure 15. Plot of: (c) excess thermal expansion coefficients against mole fraction for \{benzylalcohol (1) + phenylethanol (2)\} at the temperatures $298.15 \mathrm{~K} ; \bullet, 303.15 \mathrm{~K} ; \boldsymbol{\bullet}, 308.15 \mathrm{~K} ; \boldsymbol{\Lambda}, 313.15 \mathrm{~K} ; \bullet, 318.15 \mathrm{~K}$; .

\subsection{The Partial Molar Volume}

The partial molar volume $\bar{V}_{i}$ of each component was calculated by using the following reactions:

$$
\bar{V}_{i}=V_{m}^{E}+V_{i}^{*}+\left(1-x_{i}\right)\left(\frac{\partial V_{m}^{E}}{\partial x_{i}}\right)_{T, P}
$$

where $V_{i}^{*}$ is the molar volume of pure component i. By differentiating Eq. (8) and employing Eq. (10). $\bar{V}_{1}$ and $\bar{V}_{2}$ were computed according to the following equations:

$$
\bar{V}_{1}=V_{1}^{*}+x_{2}^{2} \sum_{i=0}^{3} A_{i}\left(1-2 x_{1}\right)^{i}-2 x_{1} x_{2}^{2} \sum_{i=0}^{3} A_{i}(i)\left(1-2 x_{1}\right)^{i-1}
$$

By using Eqs. (11) and (12) the partial molar volumes at infinite dilution were calculated and with the help of Eqs. (13) and (14), the excess partial molar volumes, $\bar{V}_{1}^{E, \infty}$ and $\bar{V}_{2}^{E, \infty}$ of each component at infinite dilution were calculated:

$$
\begin{gathered}
\bar{V}_{2}=V_{2}^{*}+x_{1}^{2} \sum_{i=0}^{3} A_{i}\left(1-2 x_{1}\right)^{i}-2 x_{1}^{2} x_{2} \sum_{i=0}^{3} A_{i}(i)\left(1-2 x_{1}\right)^{i-1} \\
\bar{V}_{1}^{E, \infty}=\bar{V}_{1}^{\infty}-V_{1}^{*} \\
\bar{V}_{2}^{E, \infty}=\bar{V}_{2}^{\infty}-V_{2}^{*}
\end{gathered}
$$

The values of $V_{1}^{*}, V_{2}^{*}, \bar{V}_{1}^{\infty}, \bar{V}_{2}^{\infty}, \bar{V}_{1}^{E, \infty}$ and $\bar{V}_{2}^{E, \infty}$ for all four binary systems at different temperatures are listed in Table 8. 
Table 8. The values of $V_{1}^{*}, V_{2}^{*}, \bar{V}_{1}^{\infty}, \bar{V}_{2}^{\infty}, \bar{V}_{1}^{E, \infty}$ and $\bar{V}_{2}^{E, \infty}$ for benzylalcohol + 1,3-propandiol, 1,3-propandiol, 1-propanol, 2-propanol and t-butanol mixtures at the temperatures from 298.15 to $318.15 \mathrm{~K}$

\begin{tabular}{|c|c|c|c|c|c|c|}
\hline \multirow{2}{*}{$T / \mathbf{K}$} & $V_{1}^{*}$ & $V_{2}^{*}$ & $\overline{\boldsymbol{V}}_{1}^{\infty}$ & $\overline{\boldsymbol{V}}_{2}^{\infty}$ & $\bar{V}_{1}^{E, \infty}$ & $\overline{\boldsymbol{V}}_{2}^{E, \infty}$ \\
\hline & \multicolumn{6}{|c|}{$\left(\mathrm{cm}^{3 *} \mathrm{~mol}^{-1}\right)$} \\
\hline \multicolumn{7}{|c|}{ Benzylalcohol (1) + 1,2-propandiol (2) } \\
\hline 298.15 & 73.325 & 90.108 & 71.925 & 88.510 & -1.320 & -1.520 \\
\hline 303.15 & 73.419 & 90.395 & 71.956 & 88.745 & -1.465 & -1.650 \\
\hline 308.15 & 73.595 & 90.809 & 72.083 & 89.016 & -1.520 & -1.749 \\
\hline 313.15 & 73.786 & 91.143 & 72.181 & 89.405 & -1.605 & -1.730 \\
\hline 318.15 & 73.971 & 91.536 & 72.295 & 89.658 & -1.671 & -1.869 \\
\hline \multicolumn{7}{|c|}{ Benzylalcohol (1) + 1-propanol (2) } \\
\hline 298.15 & 73.257 & 92.985 & 71.505 & 90.523 & -1.803 & -2.543 \\
\hline 303.15 & 73.415 & 93.418 & 71.527 & 90.690 & -1.896 & -2.670 \\
\hline 308.15 & 73.602 & 93.834 & 71.693 & 91.321 & -1.921 & -2.693 \\
\hline 313.15 & 73.786 & 94.291 & 71.856 & 91.491 & -1.951 & -2.770 \\
\hline 318.15 & 73.973 & 94.788 & 72.122 & 92.071 & -1.931 & -2.710 \\
\hline \multicolumn{7}{|c|}{ Benzylalcohol (1) + 2-propanol (2) } \\
\hline 298.15 & 73.227 & 92.276 & 72.914 & 87.958 & -0.321 & -4.337 \\
\hline 303.15 & 73.410 & 92.800 & 72.798 & 88.457 & -0.613 & -4.313 \\
\hline 308.15 & 73.593 & 93.262 & 72.907 & 89.082 & -0.795 & -4.189 \\
\hline 313.15 & 73.778 & 93.791 & 72.782 & 89.645 & -0.994 & -4.238 \\
\hline 318.15 & 73.973 & 94.318 & 72.704 & 90.339 & -1.299 & -3.989 \\
\hline \multicolumn{7}{|c|}{ Benzylalcohol (1) + 1,3-propandiol (2) } \\
\hline 298.15 & 73.225 & 103.862 & 74.028 & 102.910 & 0.796 & -0.962 \\
\hline 303.15 & 73.410 & 104.248 & 74.230 & 103.293 & 0.822 & -0.952 \\
\hline 308.15 & 73.596 & 104.640 & 74.440 & 103.783 & 0.843 & -0.857 \\
\hline 313.15 & 73.778 & 105.035 & 74.646 & 103.915 & 0.870 & -0.717 \\
\hline 318.15 & 73.965 & 105.445 & 74.872 & 104.839 & 0.899 & -0.586 \\
\hline \multicolumn{7}{|c|}{ Benzylalcohol + phenylethanol } \\
\hline 298.15 & 73.235 & 103.862 & 74.118 & 102.902 & 0.894 & -0.970 \\
\hline 303.15 & 73.419 & 104.338 & 74.239 & 103.283 & 0.920 & -0.969 \\
\hline 308.15 & 73.595 & 104.633 & 74.443 & 103.793 & 0.941 & -0.857 \\
\hline 313.15 & 73.776 & 105.035 & 74.746 & 103.914 & 0.879 & -0.736 \\
\hline 318.15 & 73.973 & 105.525 & 74.862 & 104.844 & 0.899 & -0.686 \\
\hline
\end{tabular}

As it is evident from Table 8, the partial molar volumes of benzyl alcohol at infinite dilution $\bar{V}_{1}^{\infty}$, in1,2-propandiol, 1propanol, 1,3-propandiol and 2-propanol, and the partial molar volumes of 1,2-propandiol, 1-propanol, 1,3-propandio and 2-propanol at infinite dilution, $\bar{V}_{2}^{\infty}$ in benzyl alcohol are smaller than the corresponding molar volume $V_{1}^{*}$ and $V_{2}^{*}$ of benzyl alcohol and four mentioned alcohols, respectively. This observation is consistent with an idea that the molar volume of pure components is a result of the sum of the actual molar volume plus the free or empty volume that arises from the intra-molecular self-association of pure molecules. Thus negative $\bar{V}_{1}^{E, \infty}$ and $\bar{V}_{2}^{E, \infty}$ values of benzyl alcohol + 1,2-propandiol, 1-propanol, 1,3-propandiol and, 2propanol and phenylethanol suggest the contraction in volume of the mixtures on mixing, which may be attributed to the presence of significant interactions between benzyl alcohol and 1,2-propandiol, 1-propanol, 1,3-propandiol, 2propanol and phenylethanol molecules. As a final work we modeled the experiment results by using TM and PR EOSs and results are gathered in Table 9. This is clear that the results with TM EOS are more acceptable than PR EOS. TM and PR EOS can successfully predict density and excess molar volume. And are unable to predict speed of sound.

Table 9. Average absolute deviations of the calculated densities, excess molar volumes, and speed of sound using Peng-Robinson (PR) and Tao-Mason (TM) equations of state from the measured ones.

\begin{tabular}{llll}
\hline TM EOS & AAD (density) & AAD (sound velocity) & AAD (excess volume) \\
\hline Benzylalcohol + 1, 3propandiol & 0.98 & 14.92 & 1.68 \\
Benzylalcohol + 1, 2propandiol & 0.96 & 13.18 & 1.51 \\
Benzylalcohol + t-butanol & 1.27 & 14.60 & 2.74 \\
Benzylalcohol + 1propanol & 1.09 & 16.02 & 1.41 \\
Benzylalcohol + 2propanol & 1.08 & 15.95 & 1.52 \\
PR EOS & & & 2.23 \\
Benzylalcohol + 1, 3propandiol & 1.79 & 20.05 & 1.91 \\
Benzylalcohol + 1, 2propandiol & 1.73 & 21.73 & 2.638 \\
Benzylalcohol + t-butanol & 2.52 & 19.52 & 2.25 \\
Benzylalcohol + 1propanol & 1.70 & 17.83 & 2.65 \\
Benzylalcohol + 2propanol & 1.65 & 17.51 & \\
\hline
\end{tabular}




\section{Conclusion}

This article reported the densities and speed of sound of six pure (benzyl alcohol, 1-propanol, 2-propanol, 1,2-propandiol, 1, 3propandiol, phenyl ethanol) and mixtures, at $\mathrm{T}=298.15 \mathrm{~K}$ to $323.15 \mathrm{~K}$ at $5 \mathrm{~K}$ interval and atmospheric pressure over the entire range of mole fractions using a vibrating tube densimeter. As outlined earlier all systems under study showed non-ideal behavior. This non-ideality can be explained by two factors intermolecular forces in the mixture and enhanced rigidity. The main source of intermolecular interactions comes from hydrogen bonding between the components and also interaction between $\pi$ electrons of benzene ring and proton of alkanols and instead of experimental work we can calculate thermodynamic properties.

\section{References}

[1] H. Ogawa, S. Murakami, Journal of Solution Chemistry 16 (1987) 315-326. 353.

[2] E. Jiménez, M. Cabanas, L. Segade, S. García-Garabal, H. Casas, Fluid Phase Equilibria 354180 (2001) 151-164. 355.

[3] H. A. Zarei, S. Asadi, H. Ilukhani, Journal of Molecular Liquids 141 (2008) 25-30. 356.

[4] E. Zorebski, B. Luboweiecka-Kostka, Journal of Chemical Thermodynamics 41357 (2009) 197-204. 358.

[5] E. Zorebski, M. Geppert-Rybczynska, B. Maciej, Journal of Chemical and Engineering 359 Data 55 (2010) 1025-1029. 360 .
[6] M. G. Bravo-Sanchez, G. A. Iglesias-Silva, A. EstradaBaltazar, Journal of Chemical 361 and Engineering Data 55 (2010) 2310-2315.362.

[7] M. M. Papari, H. Ghodrati, F. Fadaei, R. Sadeghi, S. Behrouz, M. N. Soltani Rad, J. Moghadasi, J. Mol. Liq. 121 (2013),180.

[8] A. Abida, S. Hyder, A. K. Nain, Collection of Czechoslovak Chemical Communications 36367 (2002) 1125-1140. 364.

[9] C. J. Wormald, C. J. Sowden, The Journal of Chemical Thermodynamics 29 (1997) 365 1223-1236. 366.

[10] C. T. Yeh, C. H. Tu, Journal of Chemical and Engineering Data 52 (2007) 1760-1767. 367.

[11] T. T. Huang, C. T. Yeh, C. H. Tu, Journal of Chemical and Engineering Data 53 (2008) 368 1203-1207.

[12] N. Sastry, NM. Vaghela, PM. Macwan, Journal of Molecular Liquids, (2013) 12-18.

[13] G. Prakash, D. KrishanKumar, Journal of Molecular Liquids, (2013) 180, 64-171.

[14] Sk. Md Nayeem, M. Kondaiah, K. Sreekanth, and D. Krishna Rao, journal of thermodynamics (2014) 13.

[15] F. Yousefi, J. Moghadasi, M. M. Papari, Ind. Eng. Chem. Res. 48, 5079 (2009).

[16] M. M. Papari, J Moghadasi, F. Fadaei, J. Mol. Liq. 165, 87 (2012).

[17] S. Sheikh, M. M.Papari, A. Boushehri., Ind. Eng. Chem. Res. 41, 3274 (2002). 